\title{
Estudos de propriedades antitermíticas de extratos brutos de madeira e casca de espécies florestais da Amazônia Central, Brasil
}

\author{
Ana Paula BARBOSA ${ }^{1 *}$, Cristiano Souza do NASCIMENTO², José Wellington de MORAIS ${ }^{1}$
}

\begin{abstract}
RESUMO
No ramo da preservação de madeira, a eficiência de compostos naturais há muito tem sido avaliada, visando encontrar substâncias que não só aumentem a vida útil de madeiras de baixa durabilidade, mas também causem menor dano ao meio ambiente. Nesse contexto, o potencial de extrativos do lenho e da casca das espécies madeireiras Pouteria guianensis, Buchenavia parviflora e Dinizia excelsa em repelir o ataque de cupins Nasutitermes sp. foi avaliado, utilizando-se como substrato a espécie Simarouba amara, de baixa durabilidade. Os extrativos foram obtidos em etanol 95\%, diluídos em solução hidroalcoólica (1 e 0,1\%), e impregnados em blocos de S. amara, segundo ASTM D1413-76. Os blocos foram montados em quadrado latino em uma plataforma e submetidos ao ataque dos cupins. O efeito das soluções foi classificado pela perda de peso dos blocos de $S$. amara. Os resultados mostraram que a maioria dos tratamentos conferiu ótima resistência à madeira de $S$. amara. O melhor tratamento foi aquele efetuado com extrativos da casca de P. guianensis, onde a perda foi somente de $10 \%$ do peso inicial dos blocos, enquanto que o menos efetivo foi aquele com extrativos da casca de D. excelsa, com perda de $70 \%$ de peso do bloco. Assim, pode-se concluir que os extrativos do lenho e da casca de $P$. guianensis e $B$. parviflora e do lenho de $D$. excelsa apresentam, a princípio, compostos com propriedades de repelência a Nasutitermes sp., e os extrativos da casca de $D$. excelsa não apresentam estes componentes.
\end{abstract}

PALAVRAS-CHAVE

Extrativos de madeira, inseticidas naturais, durabilidade de madeiras, madeiras da Amazônia

\section{Studies on the antitermitic properties of wood and bark crude extracts of forest species from Central Amazonian, Brazil}

\begin{abstract}
In wood preservation, efficiency evaluation of some natural compounds has been going on for some time, looking for substances that can be used to increase the useful life of wood species, and that are less damaging to the environment. In this context, we evaluted the potential of wood and bark extractives of the species Pouteria guianensis, Buchenavia parviflora and Dinizia excelsa in repelling Nasutitermes sp. As substrate, blocks of Simarouba amara species, of low durability, was used. The extractives were obtained in ethanol $95 \%$, diluted in hydroalcoholic solution (1\% and 0.1\%), and impregnated in a block of S. amara, according to ASTM D1413-76. The blocks were arranged on a platform in Latin square and submitted to termite attack. The effect of the solutions on termites was classified according to weight loss of the S. amara blocks. The results showed that a big majority of extractive treatments provided high resistance to S. amara wood. The best treatment was that made with the P. guianensis bark extractives, where loss was only $10 \%$ of the initial block weight. The less effective treatment was that of the $\mathrm{D}$. excelsa bark extractives, with $70 \%$ loss of block weight. Thus, we concluded that the wood and bark extractives of $\mathrm{P}$. guianensis and $\mathrm{B}$. parviflora and wood extractives of $\mathrm{D}$. excelsa present chemical compounds with repellent properties against Nasutitermes sp., while the bark extractives of D. excelsa do not.
\end{abstract}

\section{KEYWORDS}

Wood extractives, natural insecticide compounds, wood durability, Amazonian wood

\footnotetext{
1 Pesquisador do Instituto Nacional de Pesquisas da Amazônia-INPA. Av. André Araúijo, 2936, CEP 69083-000, Manaus/AM, Tel. (92)3643-3072. e-mail: morais@inpa.gov.br

2 Bolsista INPA/CNPq

* In memoriam
} 


\section{INTRODUÇÃO}

As questôes relacionadas à proteção da madeira frente a organismos xilófagos têm sido consideradas de grande relevância para o uso de espécies madeireiras de baixa durabilidade natural, pois a rápida degradação dessas espécies se constitui em um dos mais sérios problemas para o seu emprego.

No setor produtivo, o ataque desses organismos em geral pode ser prevenido pela imunização da madeira com preservantes sintéticos, sendo os produtos mais empregados à base de compostos oleosos (creosoto) ou aquosos (sais de boro, cobre, etc.) (Déon, 1989).

Contudo, alguns desses produtos são bastante prejudiciais à saúde humana e ao meio ambiente. Diante disso, a eficiência de vários compostos vegetais há muito tem sido avaliada, visando encontrar substâncias que possam ser empregadas na formulação de produtos preservantes de madeira, que apresentem não somente a eficiência como também menor dano ao meio ambiente (Logan et al., 1990; Lajide et al., 1995; Nascimento et al., 1999, 2000; Blaske \& Hertel, 2001; Li et al., 2002).

Os compostos químicos das plantas com potencial inseticida são metabólitos secundários (aleloquímicos) e o desenvolvimento atual de técnicas de isolamento e análise tem levado à identificação de um grande número de novos compostos que apresentam essa atividade, principalmente na classe dos terpenos (Champagne $e t$ al., 1992; Viegas, Jr., 2003). Dentre essa classe, os limonóides são os mais ativos com respeito ao potencial inseticida e são conhecidos como meliacinas, devido ao seu sabor amargo. Suas principais fontes são espécies das famílias Meliaceae, Rutaceae e Cneoraceae. O uso de extrativos de Meliaceae como inseticida tornou-se bem conhecido por apresentarem um amplo espectro, tanto em relação ao número de espécies de insetos afetados como em seus diferentes modos de ação. Da espécie Azadirachta indica já foram identificados cerca de 100 triterpenóides a partir das sementes, madeira, cascas, folhas e frutos (Champagne et al., 1992; Viegas, Jr., 2003).

A durabilidade da madeira está intimamente associada à sua composição química, dentre outros fatores, e também às condições ambientais do seu local de armazenamento e uso dos produtos manufaturados. Assim, é consenso que existe uma estreita relação entre a durabilidade da madeira e o tipo de extrativos ou metabólitos secundários que ocorrem nessas espécies (ZhongLuh, 1981; Fengel \& Wegener, 1984; Supriana, 1985), que pode ser explicada pelo cheiro ativo ou efeito tóxico de certas substâncias dos extrativos que atuam como repelentes ao ataque para cupins, por exemplo, ou como veneno sistêmico aos seus simbiontes (Supriana, 1985). Em madeiras tropicais, estudos realizados com espécies da regiāo dos trópicos têm constatado o efeito de extrativos quanto à sobrevivência de cupins, em testes de laboratório (Carter et al., 1983; Carter \& Camargo, 1983; Lajide et al., 1995; Blaske \& Hertel, 2001).
Assim, o presente estudo teve como objetivo avaliar o efeito de extrativos obtidos do lenho e da casca de espécies madeireiras da Amazônia Central, conhecidas como de alta durabilidade frente a cupins Nasutitermes sp.

\section{MATERIAL E MÉTODOS}

Os materiais utilizados no estudo foram extrativos obtidos do lenho e da casca das espécies madeireiras Buchenavia parviflora (tanimbuca), Dinizia excelsa (angelim-pedra), Pouteria guianensis (abiurana), extraídas de plantios da Estação Experimental de Silvicultura Tropical - (Rod. BR 174, km 45) e da Reserva Adolpho Ducke (Rod. AM 010, km 26), do Instituto Nacional de Pesquisas da Amazônia (INPA). De três árvores de cada espécie foram retirados discos de dez centímetros de espessura no diâmetro à altura do peito (DAP), que foram picotados e moídos em moinho WILEY, identificando-se as mesmas como serragem grossa. Para determinação do teor de extrativos (Norma ASTM D1107-56 e D1110-56) e de polifenóis (Costa, 1960; Vetter \& Barbosa, 1995) dessas espécies, a serragem da madeira foi peneirada, usando-se a que ficou retida na peneira de 80 "mesh". Três árvores de Scleronema micranthum (cardeiro) também foram retiradas além de uma árvore de Simarouba amara (marupá). De $S$. micranthum foram obtidos os extrativos utilizados nos testes comparativos, e de $S$. amara, os corpos-de-prova (blocos de $3,0 \mathrm{~cm}$ $\mathrm{x} 1,5 \mathrm{~cm} \times 1,5 \mathrm{~cm}$ ) que foram usados para impregnação e teste biológico.

Os extrativos da lenho e da casca foram obtidos a partir da serragem grossa das espécies, por meio de sucessivas extrações a frio com etanol $95 \%$, em frasco Mariotti, sendo o solvente retirado em rotavapor a $60^{\circ} \mathrm{C}$, e o extrato bruto sólido seco em estufa a $70^{\circ} \mathrm{C}$. As soluçôes de tratamento foram obtidas pela dissolução dos extratos brutos em mistura água:álcool (1:1), em concentração de 1 e $0,1 \%$, as quais foram impregnadas em blocos de $S$. amara, segundo a Norma ASTM 1413-76 (ASTM, 1984), permitindose um tempo de contato de $45 \mathrm{~min}$ entre a solução e os blocos, sob um vácuo de $140 \mathrm{mmHg}$. Após o tratamento, os blocos foram secos em estufa a $60-70{ }^{\circ} \mathrm{C}$ e o índice de retenção dos extratos calculado ficou entre 4,85 e $6,69 \mathrm{~kg} \cdot \mathrm{m}^{-3}$ para as espécies estudadas (10,34 a 14,71\%, em relação ao volume do bloco).

Para o teste biológico, os blocos impregnados de $S$. amara foram montados em quadrado latino em um tanque provido de plataforma de concreto e expostos ao ataque direto de uma colônia de Nasutitermes sp., em teste com preferência alimentar, utilizando-se oito repetições para cada tipo de extrativo nas concentraçôes de $1 \%$ e $0,1 \%$. Como padrão comparativo, foram usadas amostras de $S$. amara sem impregnação (amostrastestemenha) e outras tratadas com extrativos de $S$. micranthum (blocos usados como prova positiva). Após a montagem, inspeçôes diárias foram realizadas para avaliação visual do grau de ataque dos cupins. O teste foi interrompido após 60 dias, pois 
as amostras-testemunha apresentavam índice de ataque acima de $50 \%$. Então, os blocos foram cuidadosamente limpos e secos, em estufa à $100{ }^{\circ} \mathrm{C}$. A umidade dos corpos-de-prova foi controlada no inicio e no final do teste e em ambos os casos foram deixados para secar a temperatura ambiente até atingirem o peso constante. Ao final, o ataque causado pelos cupins foi avaliado pela perda de peso em percentual (PP), utilizando-se o peso inicial do bloco $\left(\mathrm{P}_{\mathrm{i}}\right)$ e o peso final $\left(\mathrm{P}_{\mathrm{f}}\right)$ como segue:

$$
P P=100 \times\left(P_{i}-P_{f}\right) / P_{i}
$$

Com esses resultados, o efeito dos extrativos sobre o ataque dos cupins foi classificado segundo os intervalos mostrados na Tabela 1, adaptado a partir de padrões para testes com madeiras (ASTM, 1984). Para avaliação mais completa, a determinação dos teores de extrativos totais e de polifenóis foi executada (Costa, 1960; ASTM, 1984; Vetter \& Barbosa, 1995).

Tabela 1 - Classes de resistência dos extrativos frente ao ataque dos cupins, em testes de laboratório

\begin{tabular}{ccc}
\hline $\begin{array}{c}\text { Perda de massa de madeira } \\
\text { tratada (\%) }\end{array}$ & $\begin{array}{c}\text { Massa residual de } \\
\text { madeira tratada (\%) }\end{array}$ & $\begin{array}{c}\text { Classificação da resistência } \\
\text { ao ataque }\end{array}$ \\
\hline $0-10$ & $90-100$ & Alta resistência \\
$11-24$ & $76-89$ & Boa resistência \\
$25-44$ & $56-75$ & Pouca resistência \\
45 & 55 & Não resistente \\
\hline
\end{tabular}

\section{RESULTADOS E DISCUSSÃO}

Os resultados encontrados mostram que extrativos do lenho das espécies $B$. parviflora, $D$. excelsa e $P$. guianensis, conferiram uma ótima resistência aos cupins à madeira tratada de $S$. amara frente ao ataque de Nasutitermes sp., pois a perda de peso das amostras foi pequena, conforme é apresentado na Tabela 2. O melhor tratamento foi aquele efetuado com os extrativos da casca de P. guianensis. Os extrativos de $S$. micranthum (lenho e casca), nos testes comparativos, não conferiram qualquer resistência às amostras de $S$. amara, confirmando resultados observados em outros estudos com a própria madeira de $S$. micranthum (INPA/ CPPF, 1991).

É relatado na literatura que a durabilidade da madeira está relacionada com o tipo e a quantidade de compostos presentes em sua composição química, como já foi abordado, e que as propriedades antitermíticas de extrativos variam com as espécies e o solvente de extração e, em alguns casos, com a concentração dos extratos (Zhong-Luh, 1981; Carter et al., 1983; Fengel \& Wegener, 1984; Supriana, 1985).

Neste trabalho, isto parece estar evidenciado, a se considerar os resultados para os teores de extrativos das espécies florestais estudadas (Tabela 3). Os cupins infestaram todos os corpos-de- prova no início dos testes, mas após dois ou três dias ignoraram as amostras impregnadas, e continuaram com o ataque às amostrastestemunha. Os corpos-de-prova impregnados com extrativos de $P$. guianensis (casca) não sofreram ataque algum pelos Nasutitermes sp. O trabalho de ataque/infestação foi observado ser mais freqüente na parte da manhã (Figura 1).

Porém, deve-se observar que a concentração padrão de solução hidroalcoólica de 1\% (amostra:solvente) para todos os extratos, com exceção do extrato de $S$. micranthum, que foi de $0,1 \%$, pode ter limitado a ação dos extrativos quanto a repelência aos cupins. Ainda assim, nota-se que um teor relativamente baixo para os extrativos de $P$. guianensis (3,1\% e 4,6\% para casca e lenho, respectivamente), foi suficiente para repelir os cupins das amostras de $S$. amara, por todo o período do teste (oito semanas completas). Resultados similares foram encontrados com cupins de madeira seca e de solo, através de testes com preferência

Tabela 2 - Classificação da resistência dos extrativos em testes com cupins Nasutitermes sp.

\begin{tabular}{|c|c|c|c|c|}
\hline $\begin{array}{l}\text { Espécie } \\
\text { madeireira }\end{array}$ & $\begin{array}{c}\text { Origem dos } \\
\text { extrativos }\end{array}$ & $\begin{array}{l}\text { Perda de } \\
\text { massa(\%) }\end{array}$ & $\begin{array}{c}\text { Massa residual } \\
(\%)\end{array}$ & $\begin{array}{c}\text { Classificação da } \\
\text { resistência ao } \\
\text { ataque }\end{array}$ \\
\hline Pouteria & Lenho & 16,0 & 84,0 & Boa resistência \\
\hline guianensis & Casca & 10,0 & 90,0 & Alta resistência \\
\hline Buchenavia & Lenho & 24,0 & 76,0 & Boa resistência \\
\hline parviflora & Casca & 14,0 & 86,0 & Boa resistência \\
\hline & Lenho & 14,1 & 85,9 & Boa resistência \\
\hline Dınızıa excelsa & Casca & 79,0 & 21,0 & Não resistente \\
\hline Scleronema & Lenho & 69,9 & 30,1 & Não resistente \\
\hline micranthum & Casca & 49,4 & 50,6 & Não resistente \\
\hline \multicolumn{5}{|c|}{ Espécie-testemunha } \\
\hline \multicolumn{2}{|c|}{ Simarouba amara } & 53,0 & 47,0 & Não resistente \\
\hline
\end{tabular}

Tabela 3 - Teores de extrativos e polifenóis ( $N^{0}$ de Stiasny) apresentados pelas espécies madeireiras estudadas

\begin{tabular}{ccccc}
\hline \multicolumn{2}{c}{ Espécie madeireira } & $\begin{array}{c}\text { Teor de extrativos } \\
(\%)\end{array}$ & $\begin{array}{c}\text { Polifenóis } \\
\left(\mathrm{N}^{0} \text { de Stiasny) }\right. \\
(\%)\end{array}$ & $\begin{array}{c}\text { Classe de } \\
\text { resistência dos } \\
\text { extrativos }\end{array}$ \\
\hline $\begin{array}{c}\text { Pouteria } \\
\text { guianensis }\end{array}$ & Lenho & 4,6 & nd & Boa resistência \\
\hline $\begin{array}{c}\text { Buchenavia } \\
\text { parviflora }\end{array}$ & Lenho & 8,2 & 2,3 & Alta resistência \\
\hline $\begin{array}{c}\text { Casca } \\
\text { Dinizia excelsa }\end{array}$ & Lenho & 14,6 & 1,9 & Boa resistência \\
\hline $\begin{array}{c}\text { Scleronema } \\
\text { micranthum }\end{array}$ & Lenca & 17,8 & nd & Boa resistência \\
\hline
\end{tabular}

Os resultados estão expressos em base de matéria seca. nd = Resultados ainda não disponíveis. 


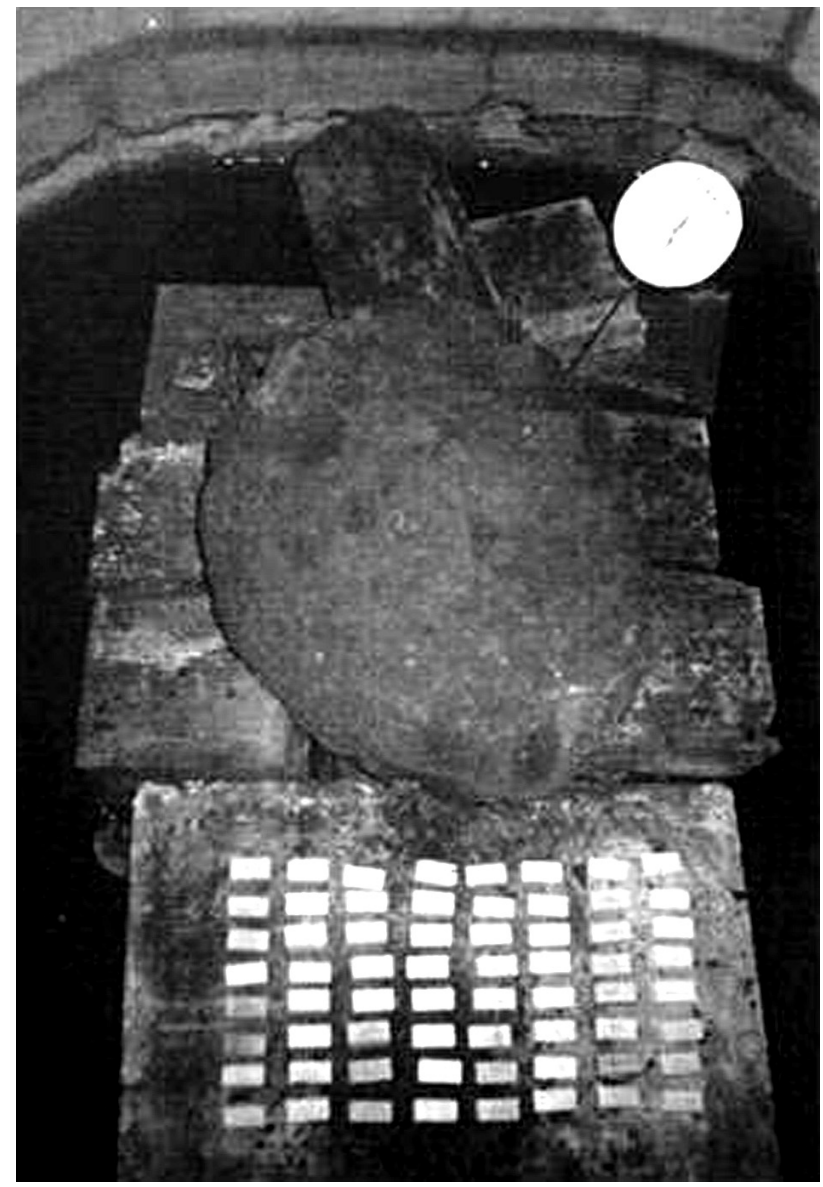

Figura 1 - Vista geral de blocos de $S$. amara tratados com os extrativos da casca e do lenho no teste com Nasutitermes sp.

alimentar, assim como testes totalmente induzidos ou de alimentação forçada (Carter et al., 1983; Carter \& Camargo, 1983; Lajide et al., 1995; Bläske \& Hertel, 2001).

Diante desses resultados, tem-se uma indicação de que os extrativos de B. parviflora, de P. guianensis e D. excelsa contêm algum composto, ou um grupo deles, que apresenta propriedades de repelência a cupins do Nasutitermes spp., dentre as condiçōes que foram estudadas. Isto pode ser particularmente procedente ao se observar nessas tabelas a relação entre a resistência apresentada pelos blocos impregnados de $S$. amara e o teor dos extrativos do lenho e da casca da espécie florestal correspondente.

No aspecto do perfil químico dos extrativos das espécies florestais, sabe-se que os extratos obtidos em etanol, como neste estudo, podem conter taninos, catequinas e outros flavonóides, esteróides, saponinas e outros terpenos, ácidos orgânicos, alcalóides e outros compostos polares (ASTM, 1984). Sobre as espécies $P$. guianensis e $D$. excelsa ainda não se tem dados, mas $B$. parviflora apresenta compostos como isoflavonas, flavonas, biflavonóides, derivados hidrolisados de taninos e triterpenóides (Li et al., 2002). Neste caso, os resultados observados demonstram que a atividade termicida ou de repelência dos extrativos poderiam ser atribuídas à atuação de alguns desses compostos, especialmente dos terpenos.

Em testes realizados para determinar a durabilidade natural de espécies florestais, utilizando-se cupins Nasutitermes sp., os ensaios com a própria madeira de P. guianensis e de Buchenavia oxycarpa (mesmo gênero de B. parviflora), apresentaram resultados classificados como altamente resistente (INPA/CPPF, 1991), o que corrobora os estudos realizados com os extrativos dessas espécies florestais.

O mesmo não se pode colocar com relação aos extrativos das espécies $S$. micranthum (lenho e casca) e D. excelsa (casca). Mesmo que $D$. excelsa tenha apresentado o maior índice de polifenóis $(8,6 \%)$, que poderia ter alguma ação quanto ao cheiro ativo, não apresentou uma ação inseticida ou repelente e, portanto, esses extrativos não parecem conter algum componente tóxico aos cupins. Neste caso, o ataque aos blocos tratados foi iniciado quase imediatamente após a montagem do teste. Vale ressaltar que o intervalo do índice de retenção para todos os extrativos nos blocos de $S$. amara ficou entre 10,3 e 14,7\% (peso de extrativo em relação ao peso do bloco).

Nos estudos subseqüentes, estão previstos o fracionamento dos extratos que apresentaram bons resultados e a realização de novos testes biológicos dessas fraçōes com cupins. Desta forma, será possível identificar-se o composto, ou um grupo deles, que apresenta as propriedades antitermíticas.

\section{CONCLUSÃO}

Diante dos resultados, pode-se concluir que os extrativos obtidos da casca e do lenho de P. guianensis e B. parviflora e do lenho de $D$. excelsa contêm, a princípio, compostos com propriedades de repelência a cupins Nasutitermes sp., enquanto que os extrativos da casca de D. excelsa e da casca e do lenho de $S$. micranthum não apresentam estes compostos, dentre as condições que foram estudadas.

\section{AGRADECIMENTOS}

Os autores expressam seus agradecimentos ao MCT Ministério da Ciência e Tecnologia e ao CNPq - Conselho Nacional de Desenvolvimento Científico e Tecnológico, pelo auxílio à pesquisa e bolsas de Iniciação Científica.

\section{BIBLIOGRAFIA CITADA}

ASTM. 1984. Annual Book of ASTM Standards. Section 4, Volume 04.09 - Wood, Philadelphia/Pa, 734pp.

Bläske, V.; Hertel, H. 2001. Repellent and toxic effects of plant extracts on subterranean termites (Isoptera: Rhinotermitidae). J. Econ. Entomol., 94 (5): 1200-1208.

Carter, F.L.; Camargo, C.R.R. 1983. Testing antitermitic properties of Brazilian woods and their extracts. Wood Fiber Sci., 15 (4): 350-357. 
Carter, F.L.; Jones, S.C.; Mauldin, J.K.; Camargo, C.R.R. 1983. Z. Ang. Ent., 95: 5-14.

Champagne, D.E.; Koul, O.; Isman, M.B.; Scudder, G.G.E.; Towers, G.H.N. 1992. Biological activity of limonoids from the Rutales. Phytochemistry, 31(2): 377-394.

Costa, A. 1960. Farmacognosia Experimental, Vol. III, Fundação Calouste Gulbekian, Lisboa.

Déon, G. 1989. Manual de Preservação da Madeira em Clima Tropical. Série Técnica 3, Yokohama: ITTO. 116pp.

Fengel, D.; Wegener, G. 1984. Wood: Chemistry, Ultrastructure, Reactions. Walter de Gruyter, Berlin and New York. 613pp.

INPA/CPPF. 1991. Catálogo de Madeiras da Amazônia. Área da Hidrelétrica de Balbina, INPA, Manaus, Amazonas. 164pp.

Lajide, L.T.; Escouba, P.; Mizutani, J. 1995. Termite antifeedant activity in Xylopia aethiopica. Phytochemistry, 40(4): 1105-1112.

Li, X.C.; Joshi, A.S.; ElSohly, H.N.; Khan, S.I.; Jacob, M.R.; Zhang, Z.; Khan, I.A.; Ferreira, D.; Walker, L.A.; Broedel, S.E. Jr.; Raulli, R.E.; Cihlar, R.L. 2002. Fatty Acid Synthesis Inhibitors from Plants: Isolation, Structure Elucidation, and SAR Studies. J. Nat. Prod., 65: 1909-1914.

Logan, J.W.M.; Cowie, R.H.; Wood, T.G. 1990. Termite (Isoptera) control in agriculture and forestry by non-chemical methods: a review. Bull. Entomol. Res., 8(3): 309-330.

Nascimento, C.S.; Morais, J.W.; Barbosa, A.P. 1999. Efeito de extrativos obtidos de espécies florestais impregnados em madeira de Simaruba amara (marupá) e submetido ao ataque de Nasutitermes sp. (Isoptera; Termitidae). Anais da VIII Jornada de Iniciação Cientifica do INPA. INPA, Manaus, Amazonas, p. 223226.
Nascimento, C.S.; Barbosa, A.P.; Morais, J.W. 2000. Efeito de extrativos obtidos de espécies florestais impregnados em madeira de Simarouba amara (marupá) e submetido ao ataque de Nasutitermes sp. (Isoptera; Termitidae). Parte II. Anais da IX Jornada de Iniciação Cientifica do INPA. INPA, Manaus, Amazonas, p. 298-301.

Supriana, N. 1985. Notes on the resistance of tropical woods against termites. IRG, Working Group Ib, Doc. No IRG/WP/1249. 9pp.

Vetter, R.E.; Barbosa, A.P.R. 1995. Mangrove Bark: A renewable resin source for wood adhesives. Acta Amazonica, 25(1/2): 6972.

Viegas Jr., C. 2003. Terpenos com atividade inseticida: Uma alternativa para o controle químico de insetos. Quim. Nova, 26(3): 390-400.

Zhong-Luh, W. 1981. The influence of extractives on wood properties and its utilization. Chemistry and industry of forest products. Chinese Soc.Forest Chem. Prop.and Eng., 1(2): 34-39.

Recebido em 06/10/2006

Aceito em 17/05/2007 
